Revue des patrimoines

$12 \mid 2009$

Le patrimoine religieuX des $\mathrm{XIX}^{\mathrm{e}}$ et $\mathrm{XX}$ siècles $-2^{\mathrm{e}}$ partie

\title{
Les souvenirs du fondateur
}

Jean Dole et Marie-Thérèse Curie

\section{Q OpenEdition}

Journals

Édition électronique

URL : http://journals.openedition.org/insitu/6570

DOI : 10.4000/insitu.6570

ISSN : 1630-7305

Éditeur

Ministère de la culture

Référence électronique

Jean Dole et Marie-Thérèse Curie, « Les souvenirs du fondateur », In Situ [En ligne], 12 | 2009, mis en ligne le 03 novembre 2009, consulté le 19 avril 2019. URL : http://journals.openedition.org/insitu/6570 ; DOI : 10.4000/insitu.6570

Ce document a été généré automatiquement le 19 avril 2019

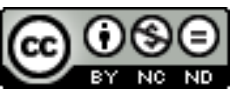

In Situ Revues des patrimoines est mis à disposition selon les termes de la licence Creative Commons Attribution - Pas d'Utilisation Commerciale - Pas de Modification 4.0 International. 


\title{
Les souvenirs du fondateur
}

\author{
Jean Dole et Marie-Thérèse Curie
}

1 J'étais vicaire à Notre-Dame de Dole ${ }^{1}$ dans les années cinquante. Cette fonction, à cette époque, me mettait en contact permanent avec les mouvements de jeunesse, ceux de l'âge d'or des "patros» et des colonies de vacances. On me demanda d'ailleurs de créer plusieurs de ces « colos » de filles (La Rixouse, les Rivières, les Perrets dans le Jura, mais aussi à l'île d'Yeu pour le diocèse). Mon bon contact avec les jeunes et la confiance de ma hiérarchie me désignèrent pour m'occuper d'une paroisse nouvellement créée à Dole, dans un quartier appelé à se développer rapidement. (fig. $\mathbf{n}^{\circ} \mathbf{1}$ )

Figure 1

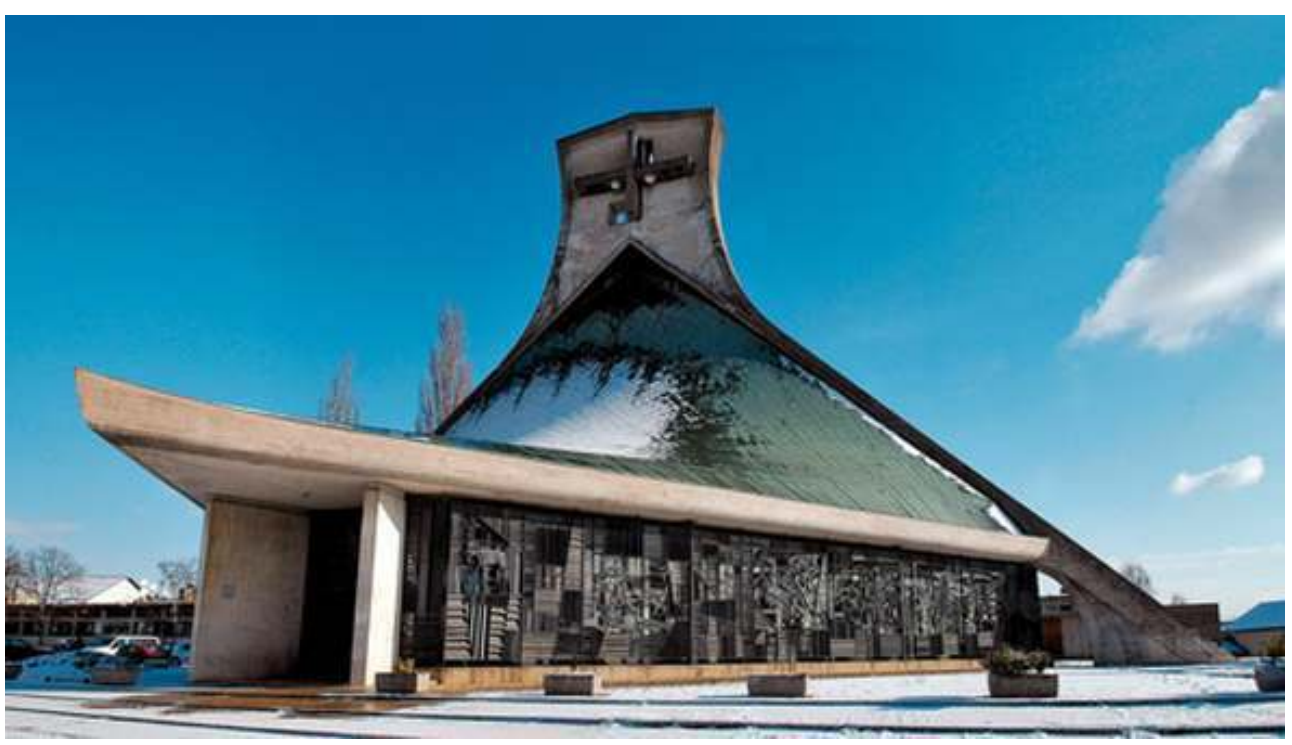

Dole (Jura), église Saint-Jean, extérieur. Phot. Henri Bertrand.

(c) Henri Bertrand. 
2 Je dois reconnaître que ces années de formation m'avaient préparé à la construction du centre paroissial Saint-Jean (église et foyer), à la suite d'un concours national d'architecture. L'édification d'une église est un acte de foi qui oblige à repenser tous les éléments pour un renouveau. C'est ainsi que l'église Saint-Jean-l'Évangéliste de Dole ${ }^{2}$ fut aussi l'église du concile Vatican II (1962-1965) qui s'est déroulé en même temps que sa construction et a marqué sa réalisation, comme l'espace centré sur l'autel, les fonts baptismaux en forme de fontaine déversant l'eau vive au puits de Jacob...

Trouver un lieu au cœur de la population était bien sûr la première tâche pour construire une église. Ce terrain, connu dans le quartier comme le « champ des sœurs ", fut cédé par la communauté Saint-Joseph-Saint-Charles de Nancy. Sur les conseils de M. Jouve (1926- ), architecte départemental et ami de l'évêque, Mgr Flusin, la décision fut prise d'organiser un concours national d'architecture, lancé le $1^{\text {er }}$ janvier 1960 . Cinq mois plus tard, M. David, architecte à Saint-Claude, et son jeune adjoint, Anton Korady, remportaient le concours devant MM. Clément-Sacquin, Brasilier (1926- ) et Novarina (1907-2002). Les lauréats firent appel à un plasticien, Maurice Calka, premier Prix de Rome de sculpture, pour créer les fonts baptismaux, mais surtout la grande grille de bronze, figurant des scènes et des textes de l'Apocalypse, qui envelopperait à l'extérieur le volume de l'église, tout en laissant passer la lumière à travers des vitres thermopanes. Les problèmes posés par la réalisation de l'édifice furent publiés dans les Annales de l'institut technique du bâtiment et des travaux publics (10 décembre 1963). La maquette et le devis estimatif furent soumis à l'approbation du conseil supérieur de l'ordre des architectes. Le choix définitif avait été guidé par des visites préparatoires, à Ronchamp ${ }^{3}$, où Le Corbusier venait d'achever l'une de ses plus fameuses réalisations, mais aussi à Fontaine-les-Grés, et même à Milan. C'est ainsi que s'imposa l'idée d'un centre paroissial, équipé d'un foyer de jeunes travailleurs, complété plus tard par un CADA (centre d'accueil pour les demandeurs d'asile). L'église paroissiale dédiée à Saint-Jean fut consacrée le 14 juin 1964 par Mgr Bertoli, nonce apostolique.

4 Cette construction n'a bénéficié d'aucun emprunt ni subvention publique. Elle a été rendue possible grâce à la générosité des fidèles, non seulement de Dole mais aussi de toutes les paroisses du diocèse, grâce à des prédications dans trois ou quatre paroisses tous les dimanches, pendant deux ans et demi! Les compagnons bâtisseurs firent des versements mensuels; chaque année était organisée une kermesse populaire qui nous aidait dans le financement. Les entreprises renoncèrent à tout bénéfice, se contentant de rentrer dans leurs frais. La construction de l'église souleva ainsi un grand courant de solidarité et de bénévolat, du plus petit au plus grand, dans le sillage de saint Jean qui a pris en charge Marie, mère de Jésus, notre mère à tous.

Après vingt ans d'activités dans la paroisse, j'ai été appelé à Lons-le-Saunier et à Villetteles-Dole pour d'autres missions. Puis, de retour à Dole et aujourd'hui âgé de 90 ans, j'ai la joie de pouvoir vivre au foyer Saint-Jean, à l'ombre de la grande flèche de béton, pointée vers le ciel.

Abbé Jean Dole 4

\section{Le sentiment d'une paroissienne}

La construction d'une église est un événement qui sort de l'ordinaire ; l'avoir vécu est un privilège. Elle est située au cœur d'un quartier qui était alors en plein développement. Mon quartier.

(fig. $n^{\circ}$ 2) 
Figure 2

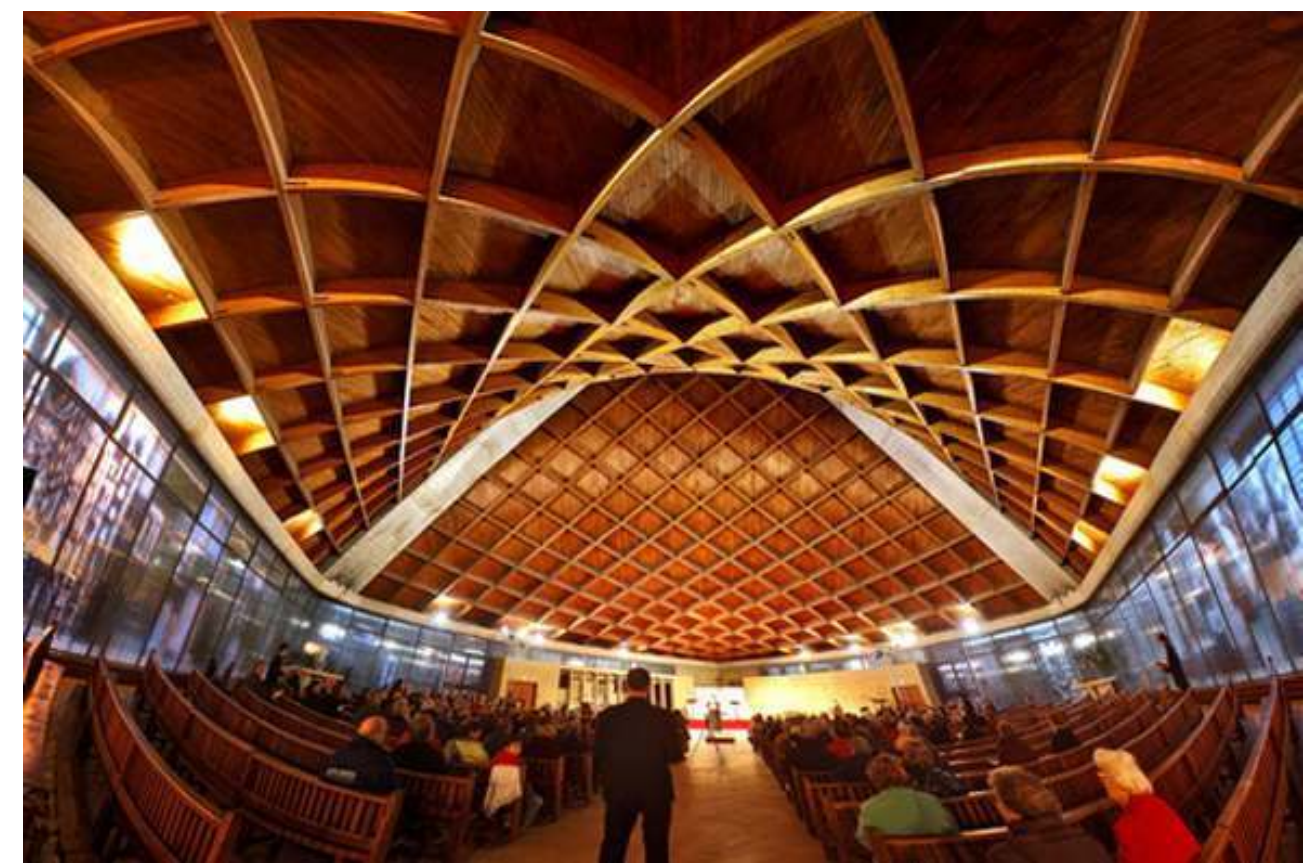

Dole (Jura), église Saint-Jean, intérieur. Phot. Henri Bertrand

Grâce à l'énergie déployée par le promoteur de cette aventure, c'est toute une ville qui s'est sentie concernée, et au-delà nombre de Jurassiens qui ont donné temps et argent pour cette réalisation, exemple unique de solidarité qui mérite d'être souligné.

7 Pour les familles du quartier, Saint-Jean était un lieu de vie qui s'offrait alors à tous (les salles du foyer, le cinéma le Régent...) Les enfants et les adolescents prirent vite l'habitude de s'y retrouver.

8 La construction elle-même fut très intéressante: depuis le creusement des fondations, très importantes, jusqu'à la sortie de terre des structures, voir s'ériger l'édifice fut un spectacle quotidien très enthousiasmant. La mise en place du grand arc, par exemple, demanda des trésors de précision qui firent l'admiration de tous. L'église présente la particularité d'être très lumineuse car elle n'a aucun mur. Je vois la superbe charpente comme une œuvre d'art magnifique, au même titre que la grille de bronze du périmètre, qui s'orne d'étranges scènes de l'Apocalypse. Pour moi, Saint-Jean est une église aussi moderne que celle de Ronchamp, par Le Corbusier, également en Franche-Comté, mais ma comparaison de non-spécialiste est peut-être un peu audacieuse...

En un mot, j'aime la beauté sobre de mon église et sa présence discrète, depuis plus de quarante ans, dans le paysage de ma ville. Sa protection par les Monuments historiques me conforte dans le sentiment qu'elle pourra rassembler encore longtemps, s'adressant à d'autres générations et dans d'autres temps.

Marie-Thérèse Curie 


\section{NOTES}

1. Voir dans la base Mérimée : notice PA00101845.

2. Voir dans la base Mérimée : notice PA39000073.

3. Voir dans la base Mérimée: notice PA00102263. Voir dans la base Mémoire: image MHR43_01700001ZA.

4. L'abbé Dole a raconté l'aventure de la création de la paroisse dans Saint-Jean. Au cœur du Jura et de la cité doloise. Dole : Les éditions de l'Est, 1994.

\section{INDEX}

Mots-clés : annales de l'institut technique du bâtiment et des travaux publics, Anton Korady, Brasilier, chapelle Notre-Dame-du-Haut, Clément-Sacquin, communauté Saint-Joseph-SaintCharles de Nancy, concile Vatican II, David, Dole, église Saint-Jean-l'Évangéliste de Dole, foyer Saint-Jean de Dole, Jean-Pierre Jouve, Le Corbusier, Maurice Calka, Mgr Bertoli, Mgr Flusin, Notre-Dame de Dole, Novarina, Ronchamp, construction d'églises

\section{AUTEURS}

\section{JEAN DOLE}

Abbé 